\title{
Study of Neural Networks and Its Application based on Fuzzy
}

\section{Adjustment}

\author{
Cong Xie ${ }^{1}$ \\ ${ }^{1}$ Guangxi University of Foreign Languages, Nanning, Guangxi, 530022 \\ ${ }^{a}$ email
}

KEYWORDS: Neural Networks; Application Cases; Fuzzy Adjustment

\begin{abstract}
With the development of fuzzy and neural network theory, the role both play in engineering applications is also growing, but both their disadvantages are also gradually revealed. The neural network has a strong ability to learn, but it is a typical "black box" model, the knowledge acquired in connection reserves the right, the user can not directly understand and use; fuzzy model of human natural language description of the problem, using fuzzy rule sets are derived, and therefore easy to understand, but it can not be expert knowledge or a large number of sample data directly into the inference rule base, and its lack of ability to learn and hard to improve itself. How to combine the advantages of both organically each other, it is increasingly becoming an issue of concern. Aiming at this problem, we made a study in the theory and application of neuro-fuzzy fusion.
\end{abstract}

\section{Introduction}

In recent years, with the development and maturation of artificial neural networks, neural networks have been widely used as a powerful computational tool for pattern recognition, artificial intelligence, classification, automation and robotics, systems engineering and other fields. Neural network consists of a large number of simple neuronal connections from each other, it is trying to replicate the computing power of biological neural networks, giving the machine has a certain cognitive organism. The neural network has strong learning ability, and is widely used in pattern recognition, intelligent control, data mining and other fields. However, the neural network learned a lot of implication in the right connection, the user can not directly understand what the network can handle the task in the end and why come to this conclusion and that, therefore, in a neural network model can not be easily understood by people form is widely accepted that a typical "black box" model. Recently, many scholars are committed by extracting knowledge in the form of symbolic rules from trained artificial neural network to change this situation. Fuzzy model is used to handle a class of fuzzy information system, which was born out of fuzzy set theory and fuzzy If-THEN rules proposed by Professor Zadeh. In reality, many of the problems are of a non-precision sub-case, in particular, no clear boundary information, and human language and behavior-related information, or due to the limitation of human knowledge and understanding rather difficult to express and clearly defined information, this information is collectively referred to as non-deterministic fuzzy information. Fuzzy systems are called a fuzzy system for information processing. Characteristics of these systems are: the behavior of the structure of the system is not clearly defined, information systems reflect the subjective human attributes and imprecise. Thus, the reasoning behavior of the system is easy to understand. Such systems with natural human language to describe the problem, 
the input-output can be either exact numeric variables can also be vague language values, it does not rely on accurate mathematical analysis of things, but from the experience and knowledge of human experts reasoning presented during the If-Then fuzzy rule set to establish a model, and thus the fuzzy control system or decision-making system based on design. However, this traditional approach there drawbacks: on the one hand, it can not automatically knowledge and experience of human experts or large amounts of real data directly into inference rule base; on the other hand, even after the establishment of the rule base, the initial fuzzy model does not necessarily reflect the true reality system, the need for effective learning method for adjusting the structural model, which includes the front and rear parts parameter optimization of fuzzy rules, under the merger and delete the partition function, reduction of redundant fuzzy rules, etc. . The fuzzy model itself lacks adaptive learning ability to improve so as to achieve the desired performance itself.

\section{Fuzzy Model and Neural Network Model}

Artificial Neural Network (ANN, Artificial Neural Network) is based on the understanding of human, artificial neural network structure can realize a function of their brain networks. It is a theoretical mathematical model of the human brain network, it is an information processing system based on imitation of brain structure and function of the network established. It is actually a complex network of interconnected large number of simple elements made of its type also varied. Since the neural network to simulate the neurons in the brain organization and function of the human brain has the basic characteristics: Learning, Memory and induction, based on artificial intelligence research to solve the logic and symbolic processing of certain limitations of artificial intelligence the study opens up new avenues. Although changes in the neural network has a strong environmental adaptive learning ability, but from the perspective of the modeling system is concerned, it uses a typical "black box" learning mode, when the study is completed, the obtained neural network input and output relationship with and can not be accepted in a manner that out, that is, it expresses information are implied, if you want to understand it is almost impossible. Fuzzy logic is based on the fuzzy thinking that humanity abstraction mechanism, described in the human way of fuzzy thinking, methods related to the macro process, while the role of intelligent form the desired excavation intelligent people, as well as analog people in this macro process . Fuzzy knowledge stored in the system rules, the rules of the fuzzy system design by experts or provided. In summary, the advantages and disadvantages of neural networks and fuzzy systems have obvious complementarities, and imitate human intelligence is both a common goal, which is an organic combination of the two creates the necessity and possibility. Therefore, the combination of the two will become a necessity. Neural networks have in learning and automatic pattern recognition strong advantage, the use of neural network technology for processing fuzzy information, enables automatic extraction of fuzzy rules and fuzzy membership functions automatically generate possible be resolved, so that the system becomes self-fuzzy Fuzzy system adaptation. Fuzzy logic technology will have the capability and higher-order information processing capability of the neural network, the neural network processing can greatly broaden the scope and capacity of information that could not only handle the precise information, but also to deal with fuzzy information.

Artificial Neurons are the basic components of the artificial neural network, each neuron can be considered people past and present model is proposed for brain nerve cells in the simplified abstraction. Although this abstract brain cells are based on knowledge-based, but in the abstract process, sometimes they have to take into account the characteristics of neural network and simulated objects and the possibility of using existing technology. Thus, the neuron mathematical model is different. Currently, the neuron model has been proposed in the hundreds, in which neuron 
model is now often used by people originally proposed is $\mathrm{M}$. P models. M-P model is a basic biological neuron simplified mathematical model, after continuous improvement, it is assumed that:

Each neuron is a multi-input single-output information processing unit;

Neuronal excitatory input points input and inhibitory inputs of two types:

There neuron output threshold characteristics, subject to the law of all or nothing: only when the sum of the input exceeds the threshold value, the neuron is activated, and when the sum of the input does not exceed the threshold, the neuron will not launch impulse;

Input and output neurons have a fixed time delay, depending on the synapses postponement:

Neurons and may have temporal characteristics and response of the whole;

When a non-variable neuron itself.

\section{Fuzzy Model Learning Strategies}

RBFN is a new neural network model that can approximate any nonlinear function at any precision, and there are no local minimum problems. RBFN typical structure is a three-forward network, wherein the hidden layer of radial basis function units take local feelings unit, radial basis function network actually consists of two layers: the hidden layer and output layer, so it contains two maps". from the input space to the hidden layer space mapping transformation is non-linear, and space from the hidden layer to the output layer space mapping transformation is linear. for multidimensional fuzzy reasoning, inference rule huge problem 1985 by Japanese scholars Takagi and Sugeno proposed T.S fuzzy reasoning model, which concluded that part of the local linear model instead of fuzzy numbers, therefore, TS model can be used to generate a small amount of complex nonlinear function rule, effectively reducing blur The number of rules.

In this paper, the typical high-end T. S fuzzy model of learning strategies, for example, start with a demonstration RBFN T-S fuzzy function equivalence of the model to start, the traditional RBFN make certain improvements, construction oriented T. S fuzzy inference model features IRBF'N (improved radial base function network), and gives the learning algorithm models (including structure learning and parameter learning), the fuzzy model of learning into IRBFN learning through the IRBFN training to achieve the fuzzy model fine-tuning purposes. The algorithm combines the advantages of fully RBFN and Ts model, using a density-based clustering algorithm to solve index number hidden layer nodes is difficult to determine the man-made problem, use a hybrid learning algorithm Fixed hidden node parameters, such that $\mathrm{T}$. Accuracy s model we expect to achieve the requirements. Finally, function approximation simulation examples can be seen that the model and corresponding algorithm is effective.

\section{The Application Cases of Fuzzy Model}

Traveling wheel is an important part of the railway vehicle, the wheels produced problems for many years has been the railway sector often met and difficult to solve. With the increase of railway traffic, and continuously improve and increase train speed rail hardness, appearing on the wheel more and more problems, and these problems are a problem in most of the wheel tread surface. "Bus rapid survey analysis and recommendations using a wheel failure condition" Academy of Railway Sciences and Sifang Locomotive Research Institute, after the whole road passenger wheel to conduct research, in 1997 the Ministry of Railways to the relevant departments of the phase of the investigation report reflected wheel tread damage phenomenon very much, and covers a wide range of complex reasons, it is difficult to solve iii1. Rear wheel tread damage occurs, it will affect the entire vehicle system, tracks and sleepers. Rail will produce periodic impact injury with a tread 
wheel running, causing damage to the rails and connecting rails and sleepers. This impact will be counterproductive to the wheel and the vehicle, causing more serious damage to the wheels, while also increasing vibration and damage to the vehicle. This not only affects the ride comfort, even more serious is the threat to road safety. For these reasons, domestic drought began research on wheel tread damage and made some tread damage diagnostic methods, including static and dynamic detection assay. A static detection contact measurement, optical measurement method; dynamic measurement method has a track circuit interruption time, eddy current, ultrasonic telemetry, impact load lean method, vibration acceleration method.

In this paper, the train wheels fault diagnosis method based on fuzzy models. First, the pre-vibration signals were recorded under normal wheels and wheel failures of rails produced by the interaction of a wheel, any change in the vibration signal will reflect the state of the wheel. Then the measured data for statistical analysis and then build confidence interval depends on the speed and frequency analysis of the state of the wheels, resulting in the failure of the wheel How to determine a priori knowledge. Next, the foregoing algorithm in the system, and establish a model for fault diagnosis and fault diagnosis based on fuzzy reasoning surfaces, making the diagnostic process becomes intuitive and easy to understand.

\section{Conclusion}

Neural networks and fuzzy models have their advantages and disadvantages, how the organic combination of the two, learning is increasingly becoming an issue of concern. In this paper, this problem is proposed learning algorithm structure and parameters of fuzzy model based on neural networks, fuzzy reasoning model is embodied in the neural network topology among the learning ability of neural network to achieve modeling and fuzzy rules in the fuzzy model optimization of the parameters, while fuzzy neural network model allows the nodes and connections with a clear meaning, so that the "black box" state of the neural network is improved, more easily understood.

\section{References}

[1] Sushmita Mitra, Member, IEEE, And Yoichi Hayashi.Neuro-Fuzzy Rule Generation: Survey in Soft Computing Framework. IEEE Transactions on Natural Networks, 2001, 11 (3): 748-768.

[2] Jyh-Shing Roger Jang. Antis. Department Of Electrical En \# Neering Andcomputerscience University California, Berkeley, CA94720.

[3] Homik K Stinchcombem White H. Multiplayer Feed forward Networks Arcuniversal Approximator. Neural Computing, 1990,2: 210-215.

[4] Kosko B, Fuzzy Systems As Universal Approximators. IEEE Transactions of Computers, 1994,43 (1 1): 1329-13. 